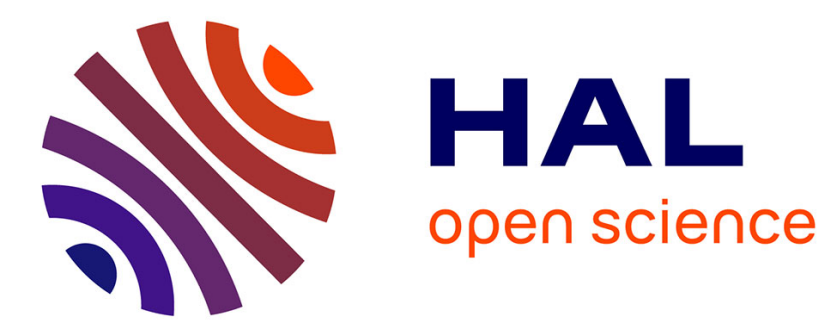

\title{
De la relation auteur-éditeur Entre dialogue et rapport de force
}

\author{
Olivier Bessard-Banquy
}

\section{To cite this version:}

Olivier Bessard-Banquy. De la relation auteur-éditeur Entre dialogue et rapport de force. A contrario.

Revue interdisciplinaire de sciences sociales, 2018, 10.3917/aco.182.0079 . hal-02521737

\section{HAL Id: hal-02521737 \\ https://hal.science/hal-02521737}

Submitted on 27 Mar 2020

HAL is a multi-disciplinary open access archive for the deposit and dissemination of scientific research documents, whether they are published or not. The documents may come from teaching and research institutions in France or abroad, or from public or private research centers.
L'archive ouverte pluridisciplinaire HAL, est destinée au dépôt et à la diffusion de documents scientifiques de niveau recherche, publiés ou non, émanant des établissements d'enseignement et de recherche français ou étrangers, des laboratoires publics ou privés. 


\title{
De la relation auteur-éditeur \\ Entre dialogue et rapport de force
}

\author{
OLIVIER BESSARD-BANQUY
}

\section{Fantasmes au harem}

La relation auteur-éditeur est l'objet de tous les fantasmes dans l'imaginaire collectif. Il n'y a rien là que de très logique puisque ce couple extraordinaire donne corps à des œuvres qui marquent en profondeur la société et que des binômes devenus fameux l'ont incarné, Proust et Gallimard, Beckett et Lindon, Gracq et Corti, et avant eux déjà Jules Verne et Pierre-Jules Hetzel ${ }^{1}$. Que serait la très noble maison Gallimard sans ses écrivains, de Gide à Le Clézio sans oublier Sartre ou Camus, Raymond Queneau et tous ceux qui ont fait le prestige ou le renom de «la banque centrale des lettres »? Inversement Céline aurait-il luimême réussi à s'imposer avec tant de tranchant sans le soutien sans faille de son éditeur Robert Denoël qui ira jusqu'à écrire une Apologie de Mort à crédit en 1936 ? Pas d'auteur sans éditeur et, de la même manière, pas d'éditeur sans auteur. L'un fait l'autre, l'un ne va pas sans l'autre. Mais cette relation cache en vérité un déséquilibre, ou plutôt une différence de nature, car si leurs intérêts sont liés, indiscutablement, leurs positions, leurs points de vue, à tout le moins, sont distincts et leurs préoccupations ou leurs obsessions à l'occasion opposées, parfois inconciliables. L'un est par nature cantonné au strict domaine de la création, l'autre est par métier dans le monde pratique de la vente, ils ne se soucient pas des mêmes choses, ils ne poursuivent pas les mêmes buts. L'un veut s'imposer dans le domaine des arts, l'autre veut réussir sur le plan des affaires ${ }^{2}$.

Au début, comme dans toutes les histoires, tout est au mieux. L'auteur, spontanément s'il s'agit d'un pur travail de création, en réponse à une commande s'il s'agit davantage d'une production sollicitée, remet un texte qui a été retenu pour publication, soit en raison de ses qualités intrinsèques, soit du fait de ses potentialités commerciales. L'éditeur a choisi son auteur, avant ou après la réalisation du manuscrit, et l'artiste en est transformé. Il a désormais «son » éditeur, un possessif à la fois charmant et source de bien des malentendus, on y reviendra, car si lui n'a qu'un éditeur, le plus souvent, le professionnel du livre, de son côté, a des dizaines voire des centaines pour ne pas dire des milliers d'auteurs. Ce n'est pas sans raison qu'un écrivain très imaginatif a un jour comparé cette situation à celle d'un sultan qui a dans son harem des femmes toutes aussi sensibles ou jalouses se déchirant son amour impossiblement exclusif. L'éditeur lui aussi a «ses» auteurs mais il ne peut matériellement pas, quand bien même il le voudrait, répondre à toutes les demandes de soins ou d'attentions que tous attendent de lui, ce qui est source de petites souffrances ou d'autant de vexations. Dans les faits, son attention est essentiellement captée par les plus importants de ses artistes sous contrat, autrement dit ceux dont sa maison dépend ; les autres n'ont droit bien souvent qu'à des égards, polis, affectueux même, sincères, mais contingentés par les nécessités de la conduite d'entreprise.

Cette relation a fait l'objet d'un livre signé de Sylvie Perez, passée par L'Express ou France Inter, qui insiste sur le caractère fort de ce lien unique, comparable en tous points à des

\footnotetext{
${ }^{1}$ Voir les très nombreuses correspondances auteurs-éditeurs publiées, notamment celles de Proust ou Céline données à la NRF grâce aux bons soins de Pascal Fouché. Un certain nombre de ces correspondances sont conservées à l'Institut Mémoires de l'édition (IMEC) et ont été abondamment évoquées dans mon livre $\mathrm{La}$ Fabrique du livre, L'Edition littéraire au XXe siècle, un ouvrage réalisé à partir des archives déposées à l'IMEC.

${ }^{2}$ Par commodité, il ne sera ici question que d'éditeurs et d'auteurs de littérature générale.
} 
relations de couple, nous dit-on ${ }^{3}$. La journaliste recourt sans fin dans son ouvrage à cette rhétorique des oscillations du cœur évoquant toute la gamme des comparaisons possibles, des lunes de miel aux divorces en passant par la paix armée ou la relative indifférence. Cette phraséologie est tentante sinon divertissante ; elle ne correspond pourtant qu'imparfaitement à la réalité, faut-il le dire. Il s'agit d'une relation sociale où des personnes distinctes ont des intérêts communs. Que cela soit une relation humaine et qu'elle se trouve compliquée du fait des attentes de l'un et de l'autre qui ne sont pas précisément les mêmes n'en fait par pour autant une relation de couple, l'un est monogame en rêve, l'autre plus que polygame par nature, on l'a dit, ce sont deux personnes qui travaillent ensemble et qui se comprennent plus ou moins, à partir de leurs attentes très variées.

L'éditeur a une importance, il est vrai, symbolique pour ne pas dire essentielle aux yeux de l'auteur ; en publiant son œuvre il est celui qui transforme le créateur du texte en écrivain, il est celui qui le fait exister aux yeux du public en homme de lettres. Pour l'éditeur, l'artiste peut aussi avoir une double importance mais elle n'est pas du même ordre ou de la même nature ; il peut tout à la fois lui apporter du crédit intellectuel par la qualité de son œuvre et tonifier le chiffre d'affaires de l'entreprise par le montant de ses ventes. Raison pour lesquelles même les maisons commerciales aiment à publier de bons textes pour redorer leur blason et même les maisons prestigieuses aiment à publier des best-sellers pour montrer qu'elles savent vendre et qu'elles ne sont pas simplement des officines de publications raffinées pour happy few. Mais l'écrivain même fameux aura toujours plus de difficulté à trouver un bon éditeur qui sache le défendre qu'un éditeur n'aura de peine à identifier puis s'attacher un auteur de qualité ; il y a peu de maisons parisiennes susceptibles de publier de bonnes plumes qui vendent et, qui plus est, avec la concentration de l'édition, l'artiste une fois fâché avec une maison du groupe Hachette n'a plus guère le choix que de passer sous une des casaques du groupe Editis, s'il ne peut être chez Gallimard. (Autrement dit, s'il a mauvais caractère ou se montre très procédurier, il peut vite se retrouver au ban de l'édition parisienne comme certains de ceux qui ont «trahi » leur maison.) Cependant qu'un éditeur tel un joueur invétéré peut toujours espérer «se refaire ». Trouver de nouveaux textes. Lancer de nouveaux auteurs. Comme René Julliard s'est relancé après guerre avant de s'imposer pleinement par Françoise Sagan ou comme Bernard Fixot a inventé Guillaume Musso après avoir perdu Marc Levy. (Dans un monde où presque deux cents millions de personnes parlent le français, il y aura toujours plus de producteurs de textes potentiels que de maisons de littérature opulentes.)

Toutes les relations entre auteurs et éditeurs sont forcément complexes où se mêlent ainsi considérations commerciales, entente intellectuelle, accords politiques, affinités électives. Certains comme Guy Debord et Gérard Lebovici ont formé un véritable duo en parfaite harmonie et les lettres d'insultes hilarantes adressées au Tout-Paris par la maison Champ libre ${ }^{4}$ sont le parfait pendant de l'œuvre hautaine ou arrogante de Guy Debord. Mais quelles que soient les ententes ou les mésententes tout est aussi rapport de force ou de pouvoir entre les deux hommes clés du système-livre - Jérôme Ferrari le rappelle dans un volume d'entretiens ${ }^{5}$ - et le même éditeur peut être distant ou lointain avec un auteur de peu d'importance et devenir soudainement très câlin avec le même devenu poule aux œufs d'or. Inversement un écrivain choyé parce que membre d'un jury littéraire peut être pour ainsi dire abandonné par sa maison comme un chien sur une aire d'autoroute s'il démissionne de ses fonctions. Autant dire que ces relations sont tout sauf uniformes; elles évoluent au gré des succès ou des ratages, et dans tous les cas il est toujours faux de vouloir les réduire ou à un

\footnotetext{
${ }^{3}$ Voir Sylvie Perez, Un couple infernal, L'écrivain et son éditeur, Paris, Bartillat, 2006.

${ }^{4}$ Voir les volumes intitulés Correspondance en deux tomes parus à Paris chez Champ libre dans les années 1970.

${ }^{5}$ Voir Jérôme Ferrari parle des Editions Actes Sud, Paris, Presses universitaires de Paris-Ouest, 2011, p. 21.
} 
simple lien d'affaires ou à une relation de couple classique, ces liens sont d'autant plus complexes qu'ils ne sont ni exclusivement professionnels ni purement personnels mais touchent à des choses qui sont essentielles pour l'auteur et tout aussi fortes pour l'éditeur quand bien même, encore une fois, il demeure quant à lui dans une dimension au moins autant économique que culturelle des choses, ce qui n'est pas le cas du rédacteur pour qui tout est avant tout intellectuel ou culturel avant d'être économique. (Car, aussi incroyable que cela puisse paraître, même des producteurs de best-sellers comme Marc Levy peuvent avoir des prétentions et se montrer très susceptibles en cas de recensions critiques acerbes.)

Les rapports ont bien sûr fort évolué entre auteurs et éditeurs depuis la période classique. Si contrairement à une légende les créateurs ont bien été souvent rémunérés dès avant les premières lois ou mesures en faveur du droit d'auteur ${ }^{6}$, il est vrai que, tant que les espoirs de carrières commerciales en boutique n'ont pu relever que du rêve, ces liens ont pu être fort distendus ou réduits à des échanges intellectuels avec les plus prestigieux des grands éditeurs-libraires-imprimeurs du passé, d'Alde Manuce aux Estienne sans oublier les Plantin ou les Elzevier. Depuis que le livre est entré dans l'ère industrielle, cette alliance est devenue stratégique, liée à des intérêts financiers potentiellement bien plus forts ${ }^{7}$. Autant dire que ce qui pouvait être par nature un compagnonnage tranquille est devenu à tout le moins une relation d'importance, volontiers passionnelle, par l'ampleur des enjeux pour l'un comme pour l'autre ${ }^{8}$.

Les sujets qui peuvent donner lieu à débats sont multiples mais il est aisé de les recenser : il s'agit pour l'essentiel de la rémunération du travail de l'auteur, de la forme à donner au texte qui sera publié et enfin de l'exploitation entrepreneuriale du livre. A cela peuvent s'ajouter des soucis d'ordre privé quand l'artiste a épousé par exemple la fille de l'éditeur - comme cela a été le cas de Frédéric Dard - mais ce sont là des situations très rares. Dans la très grande majorité des cas les points qui font problème sont la rémunération, le travail sur les textes, la commercialisation du livre.

\section{Chefs-d'œuvre et billets de banque}

La rémunération du créateur est évidemment le premier des points de discussion qui peut vite donner lieu à des désaccords. A priori pourtant il n'y a rien de plus simple, la loi de 1957 est venue fixer les choses en France et l'éditeur ne fait que se conformer aux usages consacrés par le droit: l'auteur est payé en pourcentage sur le chiffre d'affaires de l'exploitation de ses œuvres - en moyenne $10 \%$ - et, s'il peut donc négocier le montant de ce pourcentage, il n'est pas possible pour lui de se faire payer sur une autre base. A partir du montant estimé de ce qu'il pourra percevoir, une fois les comptes arrêtés, il peut certes recevoir ce qui s'appelle un à-valoir très élastiquement calculé par l'éditeur en amont, mais cette somme ne peut être appréciée qu'à l'aune de ce que pourront être les ventes et toutes les exploitations dérivées des œuvres, traductions, passages en club ou en poche, adaptations audiovisuelles et même exploitations sous diverses formes pour les plus chanceux comme $L e$

\footnotetext{
${ }^{6}$ Voir à ce sujet les travaux de John Lough (L'Ecrivain et son public, Paris, Le Chemin vert, 1987) et ceux d'Alain Viala (Naissance de l'écrivain, Paris, Minuit, 1985).

${ }^{7}$ Voir le livre d'Isabelle Diu et Elisabeth Parinet, Histoire des auteurs, Paris, Perrin, 2013.

${ }^{8}$ Il n'est que de se souvenir de la rupture spectaculaire de Camille Laurens avec POL, en raison du soutien de l'éditeur apporté à Marie Darieussecq, accusée par sa rivale de "plagiat psychique », pour prendre la mesure des choses explosives qui peuvent être liées à ces nœuds.
} 
Petit Prince que l'on retrouve à l'occasion sur des pyjamas ou des draps de bains pour enfants... ${ }^{9}$

A priori donc il n'y a rien de plus simple que de s'entendre entre auteur et éditeur, il suffit de prendre les chiffres et de voir ce que cela peut donner. Mais hélas le malentendu s'impose bien souvent car, si l'artiste est certes habitué à vivre dans un monde libéral, il entend voir juger son travail non à l'aune de sa valeur commerciale mais en raison des efforts que cela a pu lui coûter et de la qualité qu'il trouve lui-même au produit de son œuvre, une qualité parfois extrême, l'auteur n'étant pas toujours modeste. Dans notre culture du sacre de l'écrivain, le créateur est pensé comme porteur d'un génie singulier alors qu'on le sait, dans les pays anglo-saxons, il est davantage perçu comme un professionnel, un artisan qui produit ce que le public consomme. Chez nous, et quelles que soient ses ventes, l'auteur est invité à se voir comme à l'origine du livre, alors même que la majorité des ouvrages publiés sont en fait des ouvrages de commande, exception faite des lettres il est vrai (et encore, dans le domaine de l'édition industrielle, il ne reste pour ainsi dire rien de très spontané ou de très original dans les œuvres qui sont au contraire fabriquées pour être précisément conformes aux supposées attentes du public, attentes qui par définition n'ont jamais pu être établies ${ }^{10}$ ).

Les demandes extravagantes des auteurs qui vendent fort sont tout à fait logiques et compréhensibles, l'éditeur n'a qu'à faire ses comptes pour voir comment il peut y répondre sans précipiter son entreprise dans la fosse des structures en danger (car il sait que, s'il ne trouve pas à contenter son auteur, celui-ci passera à la concurrence). Les demandes tout aussi déraisonnables de ceux qui ne vendent pas sont peut-être plus surprenantes. Ainsi Nicolas Genka qui n'a presque rien publié de son vivant, sinon de petits volumes peu diffusés, n'a-t-il pas néanmoins négligé de demander à son éditeur Christian Bourgois de l'aider à acquérir un appartement rue Mouffetard dans le Ve arrondissement. Demande à laquelle l'éditeur n'a pu répondre favorablement, comme on s'en doute, quand bien même le prix au mètre carré à l'époque ne peut être comparé à ce qu'il est aujourd'hui. C'est qu'en fait plus nous vivons dans un monde libéral, plus les auteurs tiennent ce qu'ils parviennent à arracher à leurs éditeurs comme étant la vraie marque de reconnaissance de leur talent. Ainsi peut-on comprendre que même des écrivains de très bonne réputation comme Pascal Quignard aient tout fait pour décrocher un Goncourt avec stratégie pour ne pas dire avec cynisme. Pour être pleinement reconnu comme auteur aujourd'hui il faut vendre. Le prestige de l'œuvre publiée chez un grand éditeur ne suffit plus dans un monde où le président de la République peut moquer La Princesse de Clèves. Vendre ou ne pas vendre, telle est la question. Car quelle que soit son idéologie, sa conception des lettres, l'homme de plume sait qu'il vit dans un monde qui le renverra sans cesse à la réalité de ses ventes, un monde où il sera jugé à l'aune de ses réussites commerciales et non artistiques ou intellectuelles. Nombre d'auteurs contemporains n'ont-ils pas spontanément adopté une écriture ouverte, accessible, simple, à commencer par Michel Houellebecq, non par appât du gain, mais par conformisme social, parce qu'il faut une grande force de caractère pour ne pas dire une foi démesurée pour écrire contre son époque et vivre toute une vie à contre-courant ${ }^{11}$ ? (A cela s'ajoute le fait que les auteurs pensent aussi leur éditeur plus entreprenant s'il a payé cher le manuscrit, il lui faudra faire plus de promotion pour récupérer les sommes investies. Ce qui n'est pas tout à fait faux.)

\footnotetext{
${ }^{9}$ Voir à ce sujet le chapitre qu'Emmanuel Pierrat a consacré à la succession Saint-Exupéry dans Familles, je vous hais ! Les héritiers d'auteurs, Paris, Hoëbeke, 2010.

${ }^{10}$ Voir Olivier Bessard-Banquy, L'Industrie des lettres, Etude sur l'édition littéraire contemporaine, Paris, Pocket, « Agora », 2012.

${ }^{11}$ Le fameux blog d'Eric Chevillard, L'Autofictif, repris en volume à Bordeaux aux très belles éditions de L'Arbre vengeur, fourmille de remarques acides et quelque peu désespérées sur la condition d'auteur ambitieux dans un monde hyperdémocratique horizontal où les valeurs matérielles l'ont emporté sur toute autre considération.
} 
Pour être précis, avouons que bien souvent les petites maisons ne paient pas la création au motif que leur économie est trop fragile, là encore ce n'est pas faux, ou pas toujours, tout est à discuter, à débattre, c'est l'une des raisons pour lesquelles la plupart du temps, une fois révélés, les auteurs quittent les petites maisons pour celles qui sont plus solides. Toutes les vedettes de la plume qui sont restées fidèles à leurs petites maisons sans exception - Anna Gavalda au Dilettante par exemple - sont a contrario payées sans quoi en toute logique elles eussent elles aussi rejoint l'écurie Galligrasseuil. Il y a donc de très notables différences d'une maison à l'autre, selon leur taille et leur économie, et de même d'un auteur à l'autre, selon les espérances de vente - Houellebecq peut encaisser plus d'un million d'euros pour un livre quand un poète chez Fata Morgana pourra ne rien toucher -, mais dans tous les cas la rémunération ne peut être que proportionnée au potentiel de l'exploitation commerciale, il est donc aisé de trouver à s'entendre entre auteur et éditeur. En toute logique. (Encore une fois, si malentendu il y a, c'est non pas tant en raison de l'appât du gain de l'artiste qui veut sans cesse arracher toujours plus à l'éditeur, quoique, c'est surtout que le créateur voit dans cette rémunération la reconnaissance de son talent et qu'il se trouve fort vexé quand il juge cette somme dérisoire, tenant son éditeur qu'il juge mesquin pour responsable quand en fait c'est la désaffection du public qui est en cause ${ }^{12}$.)

\section{Simple correction ou standardisation?}

Il en va tout autrement dans le domaine du travail sur le texte où, comme on l'imagine sans peine, tout devient très subjectif, ouvert à tous les débats et donc à toutes les mésententes. L'auteur, encore une fois, en raison de la mythologie qui entoure sa figure, tient volontiers son œuvre pour achevée une fois le point final apporté au manuscrit et voit quelque peu abusivement l'éditeur comme un simple imprimeur, un prestataire de services qui va faire connaître ce noble travail et rémunérer l'artiste comme son talent l'exige. Le professionnel du livre tout à rebours voit le manuscrit comme le point de départ du travail éditorial, la matière première à partir de quoi, peut-être, si tout va bien, un volume pourra être donné au public, exploité commercialement. On devine dans cette divergence de vues les très nombreux désaccords qui peuvent survenir tout au long de ce processus de transformation du manuscrit en objet manufacturé.

Ainsi l'œuvre publiée, hier comme aujourd'hui, passe-t-elle par l'étape de la mise en forme éditoriale. Il ne s'agit pas tant de corriger ce qui ne va pas que d'adapter le texte au public, celui auquel l'éditeur veut s'adresser, celui qu'il vise, qu'il a repéré comme étant son public. Ce travail de l'ombre, qui peut aller du simple toilettage de surface à une réécriture complète voire une négrification du texte ${ }^{13}$, n'est souvent pas pour satisfaire l'auteur. Pour lui l'éditeur est au service de son œuvre. Il doit l'aider à porter son texte vers le lecteur. Alors que l'éditeur se pense comme au service du public et ambitionne de lui apporter quelque chose qui le satisfasse. Qui soit de la meilleure qualité possible à ses yeux, quel qu'ait pu être l'état de la copie déposée.

Ce malentendu originel est la source de tous les conflits possibles. L'éditeur n'a pas d'autre choix que de chercher à convaincre l'auteur du bien-fondé de ses remarques. Bien-

\footnotetext{
${ }^{12}$ Il est vraiment très étonnant qu'aucun chercheur ne se soit jamais penché sur la logique du droit d'auteur et la rémunération proportionnée aux ventes. Il y a consensus parmi tous pour considérer que la logique libérale est préférable aux pensions et autres opérations de mécénat ou de clientélisme de l'Ancien Régime. Pourtant, cette nouvelle logique ipso facto incite les auteurs à «travailler plus pour gagner plus », changeant radicalement la nature même de ce qui a pour nom littérature. Elle devait être ce qui était le meilleur ou le plus fin ; elle devra devenir ce qui plaît le plus, ce qui se vend le plus.

${ }^{13}$ Lire à ce sujet le très divertissant texte anonyme, Vocation nègre, Bruxelles, Labor, 2004.
} 
fondé de devoir adapter son travail aux goûts voire aux exigences du public. Mais le créateur est-il à même de se laisser gagner par les arguments de l'éditeur ? Le sacre de l'écrit dans nos sociétés, l'ivresse de soi dans la publication ne tournent-ils pas la tête de l'artiste qui sait ce qu'il veut dire et comment il veut le dire ? Est-il vraiment en état d'entendre qu'il existe peutêtre un meilleur moyen de s'exprimer ? Comment ce travail se passe-t-il ? Est-il bien accepté des auteurs? La marketisation de la littérature entraine-t-elle une dénaturation des textes plus forte aujourd'hui qu'hier? Quelle différence entre la littérature de création et la littérature de grande diffusion?

Aujourd'hui, en fait, une sorte de grande partition traverse le monde des lettres. En haut de la pyramide éditoriale domine une pléiade de grandes maisons qui de plus en plus imposent des productions standardisées. Il existe bien sûr de sensibles différences entre Grasset, Flammarion, Albin Michel, Plon ou Robert Laffont, mais une simple plongée dans le catalogue de ces anciennes maisons suffit à pressentir que plus rien ici ne relève du seul caprice de l'auteur ${ }^{14}$. Les livres publiés sont pour l'essentiel des produits qui, s'ils ne sont pas tous le fruit de commandes passées - encore que la commande, même en littérature, tende à gagner toujours plus en importance, le succès inaugural de La Bicyclette bleue de Régine Deforges ayant montré que c'était là une possible voie d'avenir ${ }^{15}-$, ne correspondent plus en rien au travail spontané de l'auteur tel que la conception romantique de l'homme de génie a pu conduire à l'imaginer. Si les grandes maisons persistent à l'occasion à révéler un ou deux auteurs sortis de nulle part, et largement médiatisés comme des hommes de plume extérieurs au sérail ${ }^{16}$, arrivés dans l'anonymat de l'envoi postal, c'est justement pour cacher l'ampleur souterraine des bouleversements du jeu éditorial standardisé, c'est pour continuer à jouir de l'image ancienne de l'éditeur révélateur de talents, démocrate sublime des lettres, refusant le texte malhabile pour ne porter au pinacle que le génie ou le talent.

Cette standardisation est le produit d'un gigantesque mais invisible travail éditorial réalisé pour calibrer le texte, pour en évacuer tout ce qui est insatisfaisant, non plus en termes littéraires, mais en termes commerciaux. Certes, sont toujours corrigées les bévues, les scories, les bourdes et autres boulettes - et personne ne peut s'en plaindre. Mais sont également retravaillées désormais toutes les longueurs, les finesses, excroissances ou fantaisies, tout ce qui peut être de nature à fatiguer ou décourager les lecteurs impatients. Plus la maison vise un large public et plus elle évacue du texte tout ce qui peut diviser plutôt que fédérer, tout ce qui peut rebuter les lecteurs les moins endurants, des consommateurs de textes, enfants de la société du zapping, peu susceptibles de se concentrer longtemps sur un écrit ardu, élaboré, complexe ${ }^{17}$. Que reste-t-il de la littérature telle que les anciens ont pu la concevoir? Rien selon les plus alarmistes des penseurs contemporains. Un récit plat, lisse, sans surprise, sans originalité. «Une littérature sans estomac » comme le dit Pierre Jourde ${ }^{18}$.

Gallimard, «la reine Victoria des lettres », dont le centenaire aura été le grand événement culturel de l'année 2011, apparaît toujours comme au-delà. Hors classe ou hors catégorie. Protégée contre toute décote par le prestige de ses publications passées. A la fois parce que les meilleurs écrivains aspirent encore à y publier aujourd'hui - c'est d'ailleurs la

\footnotetext{
${ }^{14}$ Voir le texte de la revue Décapage, n 57, Paris, Flammarion, été-automne 2017, sur l'édition de 1900 à 2000.

${ }^{15}$ Paul-Loup Sulitzer dans les années 1980 aura de même grandement popularisé la figure de l'auteur fabriqué.

${ }^{16}$ Voir encore en 2010 la révélation chez Grasset de Laurent Binet, jeune professeur de lettres en Seine-SaintDenis, auteur d'un premier roman salué par la critique et le public, $H H h H$. Grasset qui publie par ailleurs Alexandre Jardin, Yann Moix, Frédéric Beigbeder, Virginie Despentes, autant d'auteurs qui ne sont pas tout à fait des archétypes de l'artiste solitaire et surdoué travaillant dans une sorte de tour d'ivoire à une œuvre supérieure conçue pour l'éternité.

${ }^{17}$ Voir le compte rendu des débats sur l'évolution de la lecture numérique organisés par le magazine Livres Hebdo, n 821, 14 mai 2010.

${ }^{18}$ Pierre Jourde, La Littérature sans estomac, Paris, L’Esprit des péninsules, 2002.
} 
maison qui reçoit le plus de manuscrits, près de 6500 en $2006^{19}$, probablement plus de 7000 aujourd'hui, chiffre qui montre à lui seul que la NRF apparaît toujours comme le lieu premier, matriciel, fondamental, de révélation de la littérature. Et parce qu'il y a rue Sébastien-Bottin pardon, rue Gaston-Gallimard - une tradition du respect du texte et de son producteur. Que la maison au fond rechigne à trop intervenir, à trop s'engager dans le domaine du traficotage des textes. Les auteurs-stars venus d'ailleurs n'ont-ils pas eu, souvent, l'impression d'être comme en décalage, mal à l'aise par rapport au reste des auteurs-maison? N'est-ce pas sous la «Blanche» de Gide, de Sartre et Camus, que Philippe Djian a perdu le plus gros de ses lecteurs ? Mais la maison, et c'est bien normal, ne peut pour autant rester inactive dans le domaine de l'édition commerciale. Elle doit comme ses consœurs savoir produire et lancer des textes standardisés pour toucher le public le plus large et guerroyer dans des domaines où elle peut recruter de nouveaux lecteurs. N'est-ce pas d'ailleurs ce qu'elle fait avec succès quand elle publie, en "Blanche », une ex-otage de la jungle maltraitée par des guérilleros marxistes et brutaux ? Qui peut croire un instant qu'il n'y ait pas eu ici un puissant travail de mise en forme éditoriale pour aboutir à la version publiée ?

De nombreux gens de lettres depuis longtemps se sont plaints de cet interventionnisme, parfois humiliant, souvent douloureux. Marie-Odile Beauvais, auteur Grasset en guerre avec la rue des Saints-Pères, a fait un livre de son amertume, intitulé Discrétion assurée. Publiée en même temps que Yann Moix qui a bénéficié de toutes les faveurs de la maison, elle raconte comment elle a été sacrifiée par le clan Grasset, comment elle s'est trouvée livrée à ellemême ${ }^{20}$. Elle explique tout le travail que la maison lui a demandé d'effectuer sur son texte avec ce sempiternel mot d'ordre : tout faire pour ne surtout pas effrayer l'acheteur potentiel. Une sorte de lecteur médian hante désormais l'esprit des gens de maisons - une femme, la plupart du temps, plutôt citadine, jadis bonne élève en classe, vaguement curieuse - qu'il faut séduire par tous les moyens et ne surtout pas rebuter par des textes trop étranges ou trop déroutants. C'est toute la littérature comme forme d'art supérieure, parfois difficile, voire hermétique, qui est liquidée dans cette évolution éditoriale. C'est toute une mise au pas des créateurs qui s'organise dans le silence. Qui se fait presque naturellement tant les auteurs d'eux-mêmes s'adaptent aux prescriptions managériales, les intègrent sans même s'en rendre compte et produisent plus ou moins consciemment des textes calibrés pour plaire au grand public tel que les professionnels l'imaginent.

Les éditeurs, même les plus littéraires, essaient de coller aux éventuelles attentes de la clientèle ou de publier des ouvrages susceptibles de rencontrer les centres d'intérêt du public façonnés par les médias. En 1992, année des cinq cents ans de découverte de l'Amérique, en plus des monceaux de documents publiés sur l'événement, les lecteurs ont découvert pour la rentrée littéraire près d'une dizaine de romans avec Christophe Colomb pour personnage. Moins les livres sont originaux et plus les éditeurs se gênent les uns les autres en publiant trop d'ouvrages issus d'un même moule. Petit à petit nous passons d'une édition de création à une édition de fabrication, ce qui, paradoxalement - l'exemple de Christophe Colomb est à ce sujet éloquent -, réduit la variété des livres malgré l'augmentation pléthorique du nombre de titres. L'édition littéraire à l'instar des autres domaines de publication s'uniformise ou se normalise avec un fort développement des livres de commande par rapport aux œuvres libres ou spontanées. Pour un Lindon qui refuse de discuter avec Echenoz de ses soucis de romancier ${ }^{21}$, combien d'éditeurs n'hésitent plus à imposer toujours plus de contraintes à leurs auteurs pour s'assurer que le produit fini soit bien calibré pour plaire aux journalistes, aux libraires, aux jurés, aux lecteurs? Le traitement des ouvrages de création dans les grandes

\footnotetext{
${ }^{19}$ Voir l'ouvrage collectif L'Edition littéraire aujourd'hui, Bordeaux, Presses universitaires de Bordeaux, 2006.

${ }^{20}$ Marie-Odile Beauvais, Discrétion assurée, Paris, Melville-Léo Scheer, 2003.

${ }^{21}$ Voir Jean Echenoz, Jérôme Lindon, Paris, Minuit, 2001.
} 
maisons se rapproche de la façon dont sont réalisées les œuvres de grande diffusion, plus rien ou peu s'en faut n'est laissé au seul caprice de l'auteur dans l'élaboration du livre comme dans son lancement. Marie Darieussecq, quelques années plus tard, explique pourquoi, alors que son manuscrit est accepté chez Fayard, chez Grasset, au Seuil, elle préfère publier chez POL. «Aux Editions Fayard, Jean-Marc Roberts m'avait demandé de changer le début de Truismes; aux Editions Grasset on m'avait demandé de changer la fin, qui semblait trop pessimiste; aux Editions du Seuil, je ne sais plus ce qu'ils voulaient changer, mais ils voulaient modifier quelque chose! Chez Grasset ou ailleurs, lorsque l'on demande de changer la fin d'un livre parce qu'elle n'est pas assez "optimiste", ce n'est plus de la littérature, c'est du commerce. Paul a toujours pris les manuscrits comme ils étaient ${ }^{22}$.» Les éditeurs, globalement, plus que de simples passeurs, sont devenus de véritables producteurs d'œuvres dont l'auteur parfois n'existe même plus ${ }^{23}$ (comme dans la série Inca publiée chez Fixot). Pierre Jourde dans son vigoureux pamphlet, dix ans plus tard, s'emporte contre ces dérives du marketing qui n'empêchent pas un mode de vente traditionnel ${ }^{24}$. Alors que les textes, calibrés pour accrocher le public, sont trafiqués comme n'importe quel roman de la série Harlequin, ils restent promus par les éditeurs comme s'il s'agissait d'œuvres de pure création à l'ancienne. «On nous Paul-Loup Sulitzer », résume une chanson française des années $1990^{25}$.

\section{Le grand tabou du traficotage}

«Je suis venue avec un roman d'environ 250 pages », raconte une amie qui a publié un roman historique dans une des grandes maisons parisiennes. « [La maison d'édition] le prenait mais voulait un roman plus long [...]. J'ai rendu un nouveau manuscrit qui s'est [finalement] avéré trop long (le livre coûterait trop cher, m'a-t-on dit), j'ai donc dû supprimer environ 250 pages. Il y avait $[\ldots]$ dans la première partie des longueurs. Dans la seconde [...], j'ai dû $[\ldots]$ insister pour garder un chapitre auquel je tenais. Ma directrice littéraire voulait me le faire enlever prétextant que le chapitre pouvait être résumé et que cela ne servait à rien. L'idée que cela ne servait à rien est revenue assez souvent. De même l'argument: "cela ne se vendra pas si..." Ou encore : "ce n'est pas assez rapide" (mes analyses psychologiques n'avaient pas un franc succès). J'ai travaillé sur deux préparations de copie, noires de corrections et de suppressions. Certaines me semblaient justifiées (répétitions, phrases un peu longues), d'autres visaient à rendre le style plus moderne, créant alors un décalage avec d'autres passages. [...] Après les deux préparations de copie, ma directrice littéraire [m'a fait] remarquer que je n'avais pas beaucoup tenu compte de ses remarques. Nous avons repris certains points ensemble. Son discours qui, au début, était "c'est à vous de décider, c'est votre texte" est passé peu à peu à "si on veut le vendre il faut..." Pour le jeu d'épreuves, j'avais encore fait trop de corrections. Ma directrice littéraire m'a proposé de reprendre le jeu et de garder un tiers des corrections. J'avais un jour pour le faire. Pressée par d'autres travaux, incapable d'évaluer un tiers de corrections et de retravailler un texte dont j'étais saturée, je lui ai dit de choisir elle-même, comme elle me l'avait proposé. J'ignore ce qui a été gardé. De toute façon, même si j'avais encore repris le jeu d'épreuves, rien ne me prouve qu'on aurait tenu compte de mes indications. Globalement, au cours de cette bataille, [j'ai essayé] de tenir sur certains points, [j'ai cédé] sur d'autres. Le but [de la maison] était de proposer un roman

\footnotetext{
${ }_{22}^{22}$ Marie Darieussecq parle des Editions POL, Paris, Presses universitaires de Paris-Ouest, 2006, p. 43.

${ }^{23}$ « Dans l'ère industrielle du livre nourri parfois au n'importe quoi, l'universitaire Hélène Maurel-Indart plaide pour une meilleure traçabilité d'un texte [...] qui apporte le texte ? qui en donne l'idée ? qui le réécrit? qui l'amende? De nombreux livres comme chez Fixot ou Grasset sont produits par de véritables équipes anonymes » (Lire, novembre 2002).

${ }^{24}$ Pierre Jourde, op. cit.

${ }^{25}$ Voir Olivier Bessard-Banquy, op. cit.
} 
historique grand public, en fait je crois que [c'était] surtout un portrait de femme et qu'il était trop littéraire par rapport aux romans historiques habituels (c'est du moins ce que m'a dit mon attachée de presse pour justifier que je n'avais aucun article $)^{26}$. »

Ce témoignage, édifiant, peut être différemment interprété. On peut y voir la violence d'une maison qui considère le texte comme une matière première qu'il faut traficoter pour arriver à un produit vendable. On peut aussi y voir le professionnalisme d'une structure qui souhaite convaincre l'auteur de tout faire pour rencontrer au mieux son public. Dans les deux cas il ne semble pas qu'il y ait eu véritablement dialogue ou discussion pour chercher à emporter l'adhésion du signataire de l'ouvrage. Le manque de succès du livre est-il dû à cette standardisation du texte ? Ce travail a-t-il desservi l'œuvre ou l'auteur ? Il est évidemment impossible de répondre. C'est ce qui fait le charme ou la complexité des belles lettres, toute tentative de rationalisation brutale y est impossible. La lecture et l'évaluation des textes ne peuvent que rester subjectives. C'est ce qui explique que tout au long de l'histoire du livre des chefs-d'œuvre aient été refusés par les éditeurs. D'autres sauvagement dénaturés par des maisons sans scrupules.

Chacun sait que les textes ont toujours été plus ou moins l'objet d'interventions, de traficotages, de manipulations. L'imaginaire collectif a fait une large place aux rapports d'Alexandre Dumas avec son nègre principal, Auguste Maquet ${ }^{27}$. Les manipulations d'Hetzel forçant Jules Verne à retravailler ses œuvres pour respecter une morale tatillonne sont également connues ${ }^{28}$. Encore récemment Jean Lacouture, dans sa biographie de Paul Flamand, le patron du Seuil pendant près de cinquante ans, raconte comment le prix Goncourt d'André Schwarz-Bart, Le Dernier des justes, a été généreusement repris, réécrit, dans la maison $^{29}$. Il fait d'ailleurs du Seuil une maison particulièrement interventionniste : «Il ne faudrait pas beaucoup forcer la pensée de Flamand pour lui faire dire que les services de toutes natures rendus au texte de l'auteur valaient plus et mieux que bien des investissements financiers, avantages et pourcentages ${ }^{30}$. » Dans l'esprit d'un professionnel de la vieille école, le travail sur le manuscrit, la mise en forme du texte est le cœur même de l'activité éditoriale, le lieu de la plus-value. Sauf que l'on devine en l'espèce qu'il s'agit d'améliorer le texte, de le débarrasser de ses scories ou de ses lourdeurs. D'accompagner l'auteur sur la voie du perfectionnement de soi. D’un approfondissement de son écriture et de sa pensée.

C'est ce qui a peut-être changé ces dernières années : il semble que le travail ne se fasse plus dans le souci d'améliorer le texte, de l'élever, mais au contraire de le dénaturer, de l'abaisser. Ne surtout pas perdre des lecteurs est devenu le maître-mot. Cela se comprend au regard des évolutions récentes de la lecture, en raison du relatif déclin des «bibliomanes », remplacés par un ventre mou de lecteurs occasionnels ${ }^{31}$. Mais dans les faits cela conduit à débarrasser le texte de tout ce qui est en trop, de tout ce qui ralentit l'action, la progression de l'intrigue. In fine se retrouvent en librairie des livres de cent pages à peine qui semblent avoir été écrits avec peine par des auteurs de faible constitution. Dans l'un de mes travaux, Sexe et Littérature aujourd'hui, j'ai montré que les récits les plus crus publiés ces dernières années sont globalement débarrassés de toute description un tant soit peu étoffée, de tout portrait travaillé. Ne restent plus que des histoires de galipettes fébriles qui s'enchaînent comme

\footnotetext{
${ }^{26}$ Correspondance avec l'auteur.

${ }^{27}$ Voir le très bon livre à ce sujet de Gustave Simon, Histoire d'une collaboration, Alexandre Dumas et Auguste Maquet, Paris, Editions Georges Crès et Cie, 1919.

${ }^{28}$ Voir le volume collectif Pierre-Jules Hetzel, Un éditeur et son siècle, Saint-Sébastien, ACL éditions, 1988.

${ }^{29}$ Jean Lacouture, Paul Flamand, éditeur, Paris, Les Arènes, 2010, pp. 106-109.

${ }^{30}$ Ibidem, p. 188.

${ }^{31}$ Voir Olivier Donnat, Les Pratiques culturelles des Français à l'ère numérique, Paris, La Découverte-La Documentation française, 2009.
} 
autant de scènes de torture chez le marquis de Sade. Tout ce qui fait le sel des mémoires de Casanova, tous les dialogues dans les œuvres du plus célèbre des embastillés où le vice tente de convaincre la vertu des bienfaits de la nature - tout a disparu. Reste le récit raide des amours contemporaines, tristes et trash ${ }^{32}$.

On l'aura compris, autant de relations auteur-éditeur, autant de cas particuliers. Il est certes des professionnels peu interventionnistes par nature quand d'autres s'avèrent très intrusifs pour ne pas dire très directifs. Tout en cette affaire est question de sensibilité au verbe mais aussi de rapport de force. En vérité il n'y a pas un éditeur en situation mais mille manières d'être éditeur selon le poids dont celui-ci peut se prévaloir pour peser sur l'auteur. Plus ce dernier aura d'importance dans la maison et moins les équipes seront en mesure de lui faire entendre raison quand il faudra corriger le texte. (Ainsi s'expliquent les très mauvais volumes mal édités de gens fameux que l'on trouve chaque jour en librairie.) Il est possible que l'éditeur se trompe dans ses jugements, il est aussi possible que l'auteur s'entête à défendre son point de vue qui sera incompréhensible pour le lecteur. Il y a tous les jours des abus de pouvoir dans l'édition comme il y a des textes mal édités. La version publiée d'un écrit est souvent le fruit d'un entre-deux peut-être insatisfaisant, l'éditeur ayant cédé sur certains points pour mieux imposer d'autres modifications dans d'autres parties du texte, comment savoir?

En tout cas cette partie du travail éditorial reste assurément le grand non-dit de la publication, le grand point aveugle de l'édition contemporaine. (Encore récemment, dans un show très professionnel, quand l'éditeur Yves Pagès est venu présenter l'un des derniers auteurs édités de la maison Verticales au très couru Salon du livre de Bron, en 2017, il a répondu très longuement à toutes les questions posées et n'a éludé avec morgue que celle portant sur l'ampleur du travail sur le texte.) Dans notre mythologie le créateur est tout et l'éditeur n'est que le relais ou le passeur. Il n'est que de lire les petits livres très intéressants publiés par les Presses de l'université de Paris-Ouest sur les rapports auteurs-éditeurs pour prendre la mesure du tabou qui entoure le travail sur le texte. Tous, Marie Darieussecq, Philippe le Guillou, Olivia Rosenthal, Jérôme Ferrari, Vincent Ravalec, Tanguy Viel minimisent systématiquement le rôle de l'éditeur et assurent presque sans rire qu'aucun travail n'a été mené sur leurs textes ${ }^{33}$. Le plus honnête est peut-être Tanguy Viel qui reconnaît que chez Minuit il peut y avoir des annotations sur chacune de ses pages (mais que fait-il ensuite de ces demandes de corrections ou suggestions de modifications ? mystère ${ }^{34}$ ). Faut-il croire Olivia Rosenthal quand elle dit qu'elle ne se souvient plus de ce qui a été fait sur ses textes $^{35}$ ? Vraiment? Le plus cocasse, involontairement, reste Philippe le Guillou qui nie violemment que la moindre intervention sur ses textes ait pu avoir lieu avant de reconnaitre il est vrai qu'il a pu y avoir des retouches et même des chapitres coupés.. ${ }^{36} \mathrm{C}$ 'est qu'évoquer le travail sur le texte, c'est faire tomber l'auteur du firmament du génie, des hautes sphères de l'homme sans défaut qui vit dans les plis secrets de la langue, pour le faire entrer dans le monde commun des personnes peut-être douées mais non infaillibles et surtout, par nature, sans recul par rapport à l'œuvre produite. Or, ce qui a été conçu et réalisé, c'est une œuvre de l'esprit avec sa logique. L'éditeur, lui, veut un livre commercialisable. Et le chemin peut être long pour aller de l'une à l'autre.

\footnotetext{
${ }^{32}$ Olivier Bessard-Banquy, Sexe et Littérature aujourd'hui, Paris, La Musardine, 2010.

${ }^{33}$ Voir Marie Darieussecq parle des Editions POL, Philippe le Guillou parle des Editions Gallimard, Olivia Rosenthal parle des Editions Verticales, Jérôme Ferrari parle des Editions Actes Sud, Tanguy Viel parle des Editions de Minuit, Vincent Ravalec parle de ses éditeurs, tous parus à Paris aux Presses universitaires de ParisOuest entre 2002 et 2011.

${ }^{34}$ Op. cit., p. 43.

${ }^{35}$ Op. cit., p. 22.

${ }^{36}$ Op. cit., p. 16.
} 


\section{Fanfare et flop}

Le dernier des points qui peut faire l'objet d'un débat ou d'un dialogue entre l'auteur et l'éditeur est le lancement, la promotion, la commercialisation du volume. Autant de points qui relèvent pleinement des attributions du professionnel du livre comme indiqué clairement dans le contrat mais dont l'artiste ne peut accepter de se sentir dépossédé. Date de sortie, rédaction du communiqué de presse, envoi aux journalistes et aux libraires, plan de tournée en librairie, publicités, tout peut faire l'objet d'interminables discussions, l'homme de plume trouvant sans cesse que la maison n'en fait pas assez pour assurer la réussite commerciale de son œuvre quand l'éditeur à l'occasion trouve que son auteur n'est pas assez doué dans le domaine de la promotion comme Vincent Ravalec ironise à ce sujet dans son sémillant texte intitulé $L^{\prime}$ 'Auteur ${ }^{37}$. De manière fameuse, dans les bureaux, entend-on souvent la blague qui veut que, quand le livre marche, c'est grâce au talent de l'auteur, selon ce dernier, et quand il échoue, c'est la faute de l'éditeur qui n'a pas su le lancer avec les moyens idoines, toujours selon le même. En vérité, le créateur accumule aisément des griefs contre son éditeur pour la simple et bonne raison qu'il peut à bon droit toujours trouver qu'il eût été possible de faire plus ou mieux. Il oublie simplement que si l'éditeur agit ainsi pour tous ses auteurs la maison ne peut qu'être sur la paille à brève échéance car elle ne peut avoir les moyens d'assurer un lancement en fanfare pour toutes ses nouveautés.

Aussi n'est-ce pas pour de simples raisons d'argent que les auteurs peuvent parfois pour ne pas dire souvent quitter leur éditeur pour une nouvelle maison. L'écrivain peut être appelé à migrer quand il ne se sent plus ni compris ni soutenu par son éditeur, quand il a l'impression que ce dernier ne sait pas le défendre, le porter, quand il pense que son œuvre apparaitra différemment sous une autre casaque, quand il pense qu'elle sera mieux lue, mieux repérée, mieux identifiée. Mais, nous l'avons dit, le choix pour l'auteur n'est pas infini, du moins au sein de l'édition parisienne, et ceux qui ont quitté Minuit ou tel autre label de charme à taille humaine pour Galligrasseuil ont-ils vraiment été satisfaits de leur sort ? Ne vaut-il pas mieux être le roi d'une belle principauté plutôt qu'un anonyme dans un vaste pays moderne ? Plus la maison est grande et moins l'éditeur peut être disponible. Dans tous les cas, ce qui compte pour l'auteur, c'est que la maison soit tout entière à son service, pleinement engagée dans le soutien de son œuvre, prête à tout pour le faire rencontrer son public, ou plutôt ses publics tous ceux qui peuvent l'aimer. Mais l'éditeur peut-il jamais en faire assez aux yeux des gens de lettres?

On le voit, les motifs de dialogue ne manquent pas entre auteurs et éditeurs, les motifs de désaccords non plus. Certains s'en tiennent à de purs rapports professionnels comme Gracq et Corti, d'autres deviennent très soudés comme Pierre Michon et son éditeur chez Verdier, feu Gérard Bobillier ${ }^{38}$. C'est en tout cas un lien unique par l'importance de ce qui est en jeu pour l'auteur quand pour l'éditeur cela relève de plus en plus de l'opération commerciale. En toute logique peut-on dire que, là où le dialogue peut être le plus fécond, c'est à l'intérieur d'une édition dite traditionnelle où les deux parties se font pour ainsi dire une seule et même idée de ce qu'est la publication dans une optique culturelle. Plus à rebours on se rapproche du haut de l'échelle économique, plus il y a diffraction entre les investissements affectifs de l'auteur et le pragmatisme du professionnel du livre. Globalement, plus l'édition s'industrialise et plus le pouvoir est aux mains de l'éditeur qui impose sa manière de faire des livres. Si l'auteur ne s'y soumet pas, il sera remplacé par un autre. Mais encore une fois ce

\footnotetext{
${ }^{37}$ Voir Vincent Ravalec, L'Auteur, Paris, Le Dilettante, 1998 et Le Retour de l'auteur, Paris, Le Dilettante, 2009.

${ }^{38}$ Voir l'ouvrage collectif L'Edition littéraire aujourd'hui, op. cit.
} 
sont bien là des opérations chaque fois singulières et rien n'est plus risqué que de théoriser à l'infini sur des domaines qui ne relèvent que de multiples cas particuliers. Tout est toujours possible et de la rencontre improbable, inattendue, d'un écrivain d'exception et d'un éditeur de grand talent peut naître une œuvre qui marquera l'histoire des lettres comme Voyage au bout de la nuit (car le génie de l'artiste ne suffit pas, c'est ce que nous enseigne l'histoire du livre : pour qu'une œuvre s'impose, il faut un autre acteur qui s'engage sans limite pour le soutien de cette œuvre et la faire connaître et aimer du public, après avoir poussé l'auteur à lui donner la meilleure forme possible, et c'est là le rôle de l'éditeur). 
